\title{
Clinical Practice Guidelines for Occupational Therapists in the Evaluation and Treatment of Oculomotor Impairment Following Traumatic Brain Injury
}

\author{
Dana Aravich ${ }^{1} \cdot$ Laura Troxell $^{1}$ (D) \\ Accepted: 21 May 2021 / Published online: 2 June 2021 \\ (C) The Author(s), under exclusive licence to Springer Science+Business Media, LLC, part of Springer Nature 2021
}

\begin{abstract}
Purpose of review Currently, a lack of guidelines exists regarding best practices for occupational therapists (OTs) in the treatment and evaluation of oculomotor dysfunction following traumatic brain injury (TBI). Furthermore, individuals with TBI would benefit significantly from collaboration between OTs and optometrists during inpatient rehab.

Recent findings Although few articles examine interdisciplinary models of inpatient rehab care that include optometry, a recent pilot study is explored. Emerging evidence from the field of optometry supports the use of restorative approaches for oculomotor impairment in mild TBI; however, cases with moderate to severe TBI are not addressed.

Summary We describe an interdisciplinary approach involving collaboration between optometry and occupational therapy, yielding a comprehensive model to effectively evaluate and treat oculomotor impairments in those with TBI and facilitate improved performance in daily activities. We also provide guidelines useful for OTs working in settings where collaboration with optometry is not feasible.
\end{abstract}

Keywords Traumatic brain injury (TBI) - Mild traumatic brain injury (mTBI) - Oculomotor - Oculomotor impairment . Oculomotor dysfunction · Vision exercise · Occupational therapy · Occupational therapist · Optometry · Optometrist · Vision · Low vision $\cdot$ Interdisciplinary

\section{Introduction}

Individuals who have experienced traumatic brain injury (TBI) are prone to visual impairment. The visual pathways are extensive and complicated, beginning with the anterior structures of the eye and continuing through the frontal and posterior cortices of the brain [1,2]. Given the vastness of the visual system, it is estimated that up to $90 \%$ of individuals experience visual deficits following TBI $[3,4]$. The prevalence of specific visual impairment varies. Approximately $46 \%$ of TBI survivors experience visual field deficits, 40

This article is part of the Topical Collection on Brain Injury Medicine and Rehabilitation

Laura Troxell

mariottil@upmc.edu

1 Centers for Rehab Services (CRS), UPMC Eye Center Eye and Ear Institute, Low Vision Occupational Therapy Department, University of Pittsburgh Medical Center (UPMC), 7th Floor,Room 745, 203 Lothrop St, Pittsburgh, PA 15213, USA
$48 \%$ have convergence insufficiencies, $31-47 \%$ have accommodative insufficiencies, 6-9\% have diplopia, and 10-30\% have oculomotor deficits, [5, 6]. Even a mild TBI (mTBI) can result in long-term visual deficits, including photosensitivity, difficulty reading, headaches, and eye strain [7]. Any visual impairment sustained from TBI can impact an individual's ability to resume valued occupations $[8,9]$ and must be addressed by the rehab team.

According to the visual-perceptual hierarchy proposed by Mary Warren [10], effective vision allows us to make decisions about, and adapt to, our environment. This hierarchy describes a complex process in which the oculomotor system, visual acuity, and visual fields create the foundation. When damage to the oculomotor system occurs, patients are unable to fixate images on their fovea. The fovea is the central portion of the retina and contains the highest number of cone cells, allowing for detailed vision and color vision [11]. Humans rely on foveation for detailed tasks such as reading, identifying subtle contrasts in facial expressions, and visually identifying changes in walking surfaces. Damage to the fovea causes visual distortion and impairs higher levels of vision. 
This in turn impairs visual attention, scanning, pattern recognition, visual memory, and most importantly visuocognition, or the ability to use visual input to effectively make decisions and adapt to one's environment [10]. Impairments in oculomotor function can lead to a decreased ability to engage in activities of daily living, increased pain and discomfort during daily tasks, and an increased risk of falls [12•]. Identifying oculomotor dysfunction is especially complicated in the TBI population where cognitive impairment confounds the picture.

Although interruptions in the visual pathway are common following brain injury, occupational therapists (OTs) often feel unprepared or under-resourced when addressing these concerns [13], and even fewer feel prepared to specifically address oculomotor impairment [14••]. This is exacerbated by the lack of robust research and guidelines for treating these impairments $[12 \bullet, 15,16]$. We hope to provide clinicians with improved tools for understanding their role in identifying and addressing oculomotor impairment following TBI.

\section{What is the role of the interdisciplinary team in the evaluation of visual deficits?}

Proper observation of oculomotor function and subsequent intervention is essential for TBI survivors. In a typical medical model, OTs are often the first to screen TBI patients for visual impairment in the acute or subacute hospital setting, to ask for referrals to neuro-ophthalmology and optometry when applicable, and to provide interventions [17]. Unfortunately, many hospital systems do not provide clear referral pathways. Some providers rely on guidelines that recommend having a patient wait at least six months before receiving care from an optometrist to allow for time-based resolution of symptoms, while others start care immediately [18••]. Although it is possible for many oculomotor impairments to resolve naturally, it can be frustrating for patients who cannot put their life or recovery on pause, or for those who do not experience time-based symptom resolution.

Occupational therapists should use reliable and validated vision-screening tools to evaluate acute or subacute TBI patients, but few such tools exist. Ideally, when new tools are needed, they should be developed with the aid of an interdisciplinary team [19]; however, this is uncommon in practice. Vision screens need to assess eye alignment, convergence, saccades, smooth pursuits, visual fields, distance, intermediate, and near acuity, and contrast sensitivity $[17,19]$. One existing tool currently underutilized by OTs is the Brain Injury Visual Assessment Battery for Adults (biVABA), which assists the evaluation and treatment of functional visual impairments $[20,21]$. The biVABA contains a number of validated and reliable subtests for those with TBI. These subtests address visual acuity, contrast sensitivity, visual field, visual attention, eye dominance, diplopia testing, pupillary response, and binocular eye movements. They should be paired with functional observations of the patient to create a more holistic evaluation and to aid in intervention planning [17].

All vision assessments should also pair functional observation with patient-centered complaints, utilizing a "look and listen" approach [17]. Behaviors indicative of visual impairment include consistently tilting the head while viewing objects, shutting one eye or squinting during activities, excessive blinking, complaints of eye fatigue or headache, agitation, low frustration tolerance, sensitivity to light, reports of swirling or moving print, and eyes turning in or out when viewing objects [22]. The practitioner must ask the patient or family member explicit questions regarding changes in a patient's visual symptoms as patients often under-report visual impairments without pointed questions [23]. The Brain Injury Vision Symptom Survey (BIVSS) is a validated and reliable tool that clinicians can quickly administer to identify any visual symptoms a patient may be experiencing [24]. Unfortunately, there is a lack of occupation- or performance-based assessments that have good validity or reliability for the evaluation of functional vision impairments. One study found that the number of visual impairments identified by the OT correlated to the number of functional impairments in the patient, demonstrating the importance of occupation-based observations [19].

The cognitive demands of standardized vision tests, specifically sustained attention, are often too challenging for a TBI survivor to complete, further complicating the picture [17]. Occupational therapists are experts in activity analysis and understand both the visual and cognitive demands associated with tasks. They should therefore work closely with optometrists to help determine acceptable modifications for vision tests. For example, a high-contrast distance acuity test may be successfully completed by a patient with aphasia through the use of forced-choice questions, or a patient with neglect may benefit from having a visual anchor placed on the chart [25].

It is important to obtain an ocular history during the visual screen, including if a patient wore corrective lens prior to their injury and how current their corrective lens prescription is [17]. The patient may have lenses that correct for preexisting oculomotor impairments such as presbyopia or strabismus, and without the appropriate eyewear, false positives for oculomotor dysfunction may occur. Patients and families need to be educated and encouraged to bring in appropriate eyewear for the hospital stay [26]. Even an uncorrected visual acuity of 20/40 can negatively impact neuropsychological testing, and potentially indicate a cognitive impairment when the test results are actually due to a visual impairment [27]. In addition, TBI survivors often complain that their recent prescriptive lens no longer "work" or are blurry. An optometry 
evaluation is warranted to determine the cause and treatment. One study found that 18 to $31 \%$ of patients required adjustments in their refraction for glasses following acquired brain injury, and in some cases, completely resolved the visual complaints [28]. An oculomotor impairment may also be present if a disparity between the patient's near acuity and distance acuity is observed, and a referral to optometry or ophthalmology should be sought [17]. These situations highlight the need for a team approach that includes optometry.

\section{Occupational therapists should provide occupation-based interventions rather than restorative vision exercises}

Currently, limited research supports the use of vision therapy following TBI [12•] [16•]. However, much more research is being conducted. A survey conducted in 2019 found that $86 \%$ of vision therapy studies were conducted in the past five years [16•]. To date, optometrists have completed most of the research regarding the treatment of oculomotor dysfunction in TBI survivors [16•]. However, few have utilized an interdisciplinary approach, limiting the generalizability of these studies to most inpatient rehab settings.

Typically, optometry completes a thorough vision evaluation and prescribes appropriate visual exercises for the patient. The vision exercises are often conducted by OTs under the supervision of the optometrist and can include Brock String, Hart Accommodation Charts, and eye push-ups [28]. Although some OTs have utilized this model without consultation with optometrists, caution should be taken as this is outside the domain of occupational therapy [29]. As highlighted by the American Occupational Therapy Association's (AOTA) Occupational Therapy Code of Ethics, OTs must be mindful of the tenets of professional integrity, responsibility, and accountability, which emphasize the importance of being aware of one's own role and that of other professionals when creating an effective team [30]. Optometrists are experts in addressing binocular vision. This area of practice is complicated. We advise OTs to work closely with optometrists and strongly advocate for optometrists' involvement in the TBI team. OTs should only provide occupation-based interventions to address functional vision rather than visual exercises [15].

Effective vision therapy typically requires four to twelve weeks to complete with an average of 30 to $60 \mathrm{~min}$ per session [12•]. This intensity can only be readily incorporated into OT sessions if functional activities are utilized rather than strict vision exercises to ensure that occupation-based goals are met. This is possible if optometrists and OTs work together to translate patient-specific needs into functional programs; for example, to address convergence or accommodation deficits, a patient could look at a wall calendar and copy appointments onto a piece of paper [14••, 19].

Evaluation for refractive errors and prescription of devices for oculomotor dysfunction by optometry is a crucial component of treatment following TBI that involves vision loss. One study [31] found that $78 \%$ of patients post-concussion had changes in their refraction and that $31 \%$ had resolution of visual symptoms with new prescriptive glasses alone. Without current and accurate corrective lenses, vision exercises are a waste of therapy time, frustrating for patients, and can cause headaches, fatigue, and mistrust in the clinician.

\section{What compensatory methods can improve functional vision following oculomotor impairment?}

Although most oculomotor impairments from TBI resolve without intervention within six months [32], patients experience visual challenges in the interim that limit their daily performance and negatively impact their rehabilitation potential. There are many compensatory interventions that OTs can provide to improve patients' abilities to engage in daily activities. It is estimated that $80 \%$ of OTs utilize some compensatory techniques when providing intervention for oculomotor deficits [33]. Many of these compensatory techniques originate in low vision rehabilitation and can be effective for symptom management and increasing functional vision for those with TBIs as well.

Partial occlusion is a quick and easy option for managing diplopia without the negative impact associated with full occlusion. Ideally, partial occlusion uses the least amount of opaque tape necessary to block binocular input. Tape is typically placed on the lens of the patient's glasses over the nondominant eye, either using a nasal- or spot-taping pattern [34]. This is preferable to full occlusion because full occlusion increases a patient's risk of falls by blocking peripheral vision during dynamic tasks, and impairs depth perception, or stereopsis, during daily tasks $[17,35]$. More recent findings further indicate that full occlusion can increase the risk of new spatial neglect from the Sprague Effect [21].

There are also many environmental compensatory techniques for improving vision following TBI that OTs can utilize. Lighting sources may be modified to minimize glare by adjusting the direction or quality of the light [36]. This includes having patients wear wide-brimmed hats when outdoors, dimming electronic screens, and being cognizant of direct lighting sources such as windows or indirect lighting sources such as glossy finishes on furniture. Overhead fluorescent lighting should generally be avoided. The intensity of the light and the strobing effect are often too harsh for those with TBI and can cause discomfort and headaches [37]. It is, however, important to mention that lighting preferences are often personal. Whenever possible, therapists should ask the client if direct or indirect sources of lighting 
are causing discomfort [36]. Colored glare filters can also be used to increase a patient's comfort. Approximately $76 \%$ of individuals with mTBI had photophobia, and $85 \%$ of these individuals experienced relief when using one or more colored glare filters [37]. Although glare filter color preferences are individualized, one study found that green, blue, red, and purple glare filters are the most popular for symptom mitigation [37].

In general, while TBI patients may prefer softer light, they still need appropriate task lighting to maximize their visual potential. When task lighting is of the optimal brightness and temperature, and is positioned to prevent glare or shadows, it maximizes the contrast of the target object. This essentially magnifies the text and makes it appear clearer [38]. As with glare filters, optimal lighting preferences are individualized. The Lux IQ $\mathrm{IQ}^{\mathrm{TM}}$ is a simple lighting assessment tool that provides practical and objective recommendations for an individual's lighting needs. Other ways to decrease the visual burden include increasing the contrast of everyday objects. Simple changes such as having a patient use a white cup when pouring black coffee or placing a contrasting, colored seat cover on a black wheelchair will decrease problems resulting from oculomotor dysfunction such as impaired depth perception or blurred vision.

A primary function of oculomotor control is accommodation, or the ability to focus the eyes on near objects and tasks. This process requires a complex integration of vergence of the eyes, thickening of the lens, and pupillary constriction [39]. Near vision tasks such as reading are especially difficult when accommodative dysfunction is present. Enlarging text reduces the amount of oculomotor control a patient must use to fixate the object on their fovea and lessens the visual burden [17]. Again, interventions should be individualized to a patient's specific needs. The MNRead, a functional reading assessment that determines a patient's critical print size (the optimal text size that can be read with ease and fluency), can be useful in this context [40]. With this knowledge, appropriately sized text can be used during functional activities.

Optimizing the visual environment is essential. Relative size or relative distance magnification can also be used to compensate for decreased oculomotor control [41]. For example, a patient may need to sit closer to their TV to watch the news or get a larger TV to watch their favorite show. Optometrists must evaluate and prescribe the appropriate power, or diopters, of magnification for optical devices like hand-held magnifiers, and ideally collaboration with optometrists will be possible in all steps of the rehabilitation process. When this is not feasible, OTs can independently utilize non-optical magnification devices with patients. These include readily available electronic devices including tablets and smart phones. Numerous solutions are available to consumers through built-in accessibility features or through the use of magnification apps.

Humans rely heavily on visual input as it is the most efficient way to obtain information about the environment. Even when vision is severely impaired, humans will still attempt to use their remaining vision [17]. Occasionally, however, patients need alternative ways to access print materials for energy conservation or due to significant visual impairment. It is then appropriate to utilize auditory sources during daily activities such as optical character recognition (OCT) software or audiobooks. Many free or low-cost apps like Seeing AI or the National Federation of the Blind (NFB) Newsline can address these needs.

Finally, when collaboration with optometry is possible, another common compensatory strategy for vergence issues is the use of prisms. Vergence issues are common following TBI, and prisms are the first line treatment to restore binocularity [42]. They are effective in treating 64\% [43] to $80 \%$ [44] of patients with strabismus. Optometrists must evaluate the patient and prescribe the appropriate power and placement of the prism on the lens. Fresnel press-on prisms are a common intervention for diplopia management from cranial nerve palsies. Both the placement on the lens and the power of the prism can be modified as the extra-ocular eye control improves throughout recovery [45, 46], creating a cost-effective intervention which significantly improves quality of life.

\section{Conclusion}

Despite the evidence, much of it recent, that underlies the guidelines we present here, more research is still needed to determine the best collaborative practices for OTs and optometrists when addressing oculomotor deficits following TBI [15]. A recent survey found that very few OTs currently use evidence-based treatment for addressing oculomotor deficits [14••], and fewer than $80 \%$ of OTs polled in an earlier survey felt competent to manage such patients [47]. In addition, many limitations exist in completed studies, including small sample sizes, a lack of controls, and a lack of well-described patient populations or interventions [16•]. One pilot study, a small, randomized control trial, compared an oculomotor vision exercise protocol with an activity-based standard of care for improving oculomotor function in patients with TBI [48••]. Both groups received an evaluation from an optometrist and treatment from OTs. Both groups also made improvements during their four-week intervention. Although this study is promising, we advise OTs to proceed with caution as the OTs involved in this study had frequent guidance from optometry, worked with patients who had inpatient rehab stays of at least four weeks, and presumably had expert content knowledge to appropriately guide interventions. In addition, optometry was able to evaluate prescriptive lens needs and provide updated corrected lens when needed to the patients, which is uncommon in many facilities. Further research is necessary to determine if recovery was due to the natural resolution of deficits over time or to intervention. 
Clinicians also need to consider that vision is intricately intertwined with cognition, especially in patients with TBI. A small number of rigorous studies support the use of restorative practice and oculomotor-based vision therapies implemented by optometrists for oculomotor impairments [49]. However, these studies solely focus on mTBI; traditional vision therapies may not be effective for individuals with moderate or severe TBIs [49]. Up to $65 \%$ of individuals experience long-term cognitive impairment following moderate to severe TBI [50]. The decreased efficacy of vision therapies in those with more severe TBI may be due to a mismatch between the cognitive demands of traditional vision exercises and the cognitive reserve that patients have post-TBI. Further research is needed to elicit the impact of cognition on functional vision and best treatment practices.

Unlike the motor system, neuroplasticity of the visual system is highly dependent on function, context, and meaning [51]. Studies have shown that optimal neuroplasticity occurs during context-based learning rather than rote exercise [52-54]. Functional contexts enhance neural connections and recovery [52]. Furthermore, learning in one context may not always transfer to another. For example, completing eye push-ups may not translate to a patient's ability to read the small print on a medicine bottle. Current research supports the use of functional, contextbased approaches for improved cortical reorganization and outcomes in motor relearning for TBI survivors [53•, 54], but has rarely been applied to vision-based recovery, especially for those with more severe TBIs. Occupational therapists are well-suited to further explore the importance of context-based and functional approaches as they are experts in task analysis and regularly address both vision and cognition during meaningful activities. When the expertise of optometrists is paired with OTs, it seems possible that improvements in functional vision will be realized for a range of TBI survivors.

Furthermore, continued advocacy for the inclusion of optometry in the inpatient neuro-rehabilitation team is necessary. Keilty et al. $[18 \cdot \bullet$ piloted a model of care that overcame many of the barriers inpatient hospitals face regarding true interdisciplinary collaboration with optometry. This pilot used a hybrid model where inpatient OTs screened patients for functional vision impairments and consulted with an optometrist via telemedicine. The optometrist and OT worked together to select and modify appropriate vision tests, with the optometrist working remotely and the OT working with the patient in person. The optometrist provided recommendations for the OT to implement, including guided placement of Fresnel prisms. This program resulted in a significant increase in access to optometry care. Prisms were trialed with $49 \%$ of patients presenting with strabismus, and those patients had a $94 \%$ acceptance rate of the prism. Of note, this pilot began before the COVID-19 pandemic and was extended into it, demonstrating its adaptability in meeting patient and clinician needs during trying times. We propose wider implementation of such an approach to address patient needs creatively, quickly, and effectively.

In summary, evaluating and treating visual dysfunction in the TBI population is complicated and requires a team approach. Brain injury rehabilitation needs to advocate for the inclusion of optometry in the interdisciplinary team to provide comprehensive care for TBI patients. Careful observation of functional behavior and explicit questions regarding a patient's visual symptoms is necessary for evaluating functional vision. Although the identification and treatment of visual deficits typically falls within the domain of OT and optometry, the functional implications of oculomotor dysfunction are pertinent to all healthcare professionals. It is important to be aware of one's professional and personal limits regarding the treatment of vision impairments and to seek further education when appropriate. In general, occupational therapists should implement occupation-based interventions rather than restorative vision exercises to address postTBI oculomotor impairment. Compensatory strategies should also be implemented to optimize functional vision. Novel care delivery models can be an effective way to incorporate collaboration between optometry and OT. Further research needs to be completed to determine the relevance of context-based approaches for improving oculomotor dysfunction in a range of TBI survivors, especially when using an interdisciplinary approach. Although there is still much to be learned in the treatment of oculomotor impairment in those with TBI, there are many existing strategies available to OTs working to improve the functional vision in those with TBI, and emerging evidence strongly suggests that increased collaboration with optometry will further improve outcomes in this population.

Acknowledgements A special thank you to Holly Stants, MS, OTR/L, SCLV, CLVT for her support throughout this project.

\section{Declarations}

Conflict of Interest None of the authors has any potential conflicts of interest to disclose.

Human and Animal Rights and Informed Consent This article does not contain any studies with human or animal subjects performed by any of the authors.

\section{References}

Papers of particular interest, published recently, have been highlighted as:

- Of importance

- Of major importance

1. Singman EL. Automating the assessment of visual dysfunction after traumatic brain injury. Medic Instrument. 2013;1(1). 
2. Kelts EA. Traumatic brain injury and visual dysfunction: a limited overview. NeuroRehabilitation. 2010;27(3):223-9.

3. Ciuffreda KJ, Kapoor N, Rutner D, Suchoff IB, Han ME, Craig S. Occurrence of oculomotor dysfunctions in acquired brain injury: a retrospective analysis. Optometry. 2007;78(4):155-61.

4. Jacobson S, Marcus EM. Neuroanatomy for the Neuroscientist. 2nd ed. New York: Springer; 2011.

5. Cockerham GC, Goodrich GL, Weichel ED, Orcutt JC, Rizzo JF, Bower KS, et al. Eye and visual function in traumatic brain injury. $\mathrm{J}$ Rehabil Res Dev. 2009;46(6):811-8.

6. Suchoff IB, Kapoor N, Ciuffreda KJ, Rutner D, Han E, Craig S. The frequency of occurrence, types, and characteristics of visual field defects in acquired brain injury: a retrospective analysis. Optometry. 2008;79(5):259-65.

7. Magone MT, Kwon E, Shin SY. Chronic visual dysfunction after blast-induced mild traumatic brain injury. J Rehabil Res Dev. 2014;51(1):71-80.

8. Greenwald BD, Kapoor N, Singh AD. Visual impairments in the first year after traumatic brain injury. Brain Inj. 2012;26(11):133859.

9. Warren M. Evaluation and Treatment of Visual Deficits Following Brain Injury. In: Pendleton HM, SchultzKrohn W, editors. Pedretti's Occupational Therapy: Practice Skills for Physical Dysfunction. St. Louis: Elsevier Mosby; 2013. p. 590-630.

10. Warren M. A hierarchical model for evaluation and treatment of visual perceptual dysfunction in adult acquired brain injury, Part 1 . Am J Occup Ther. 1993;47(1):42-54.

11. Mogk LG. Eye Conditions That Cause Low Vision in Adults. In: Warren M, Barstow EA, editors. Occupational Therapy Interventions For Adults With Low Vision. Bethesda: American Occupational Therapy Association/AOTA Press; 2011. p. 27-46.

12. Watabe T, Suzuki H, Abe M, Sasaki S, Nagashima J, Kawate N. Systematic review of visual rehabilitation interventions for oculomotor deficits in patients with brain injury. Brain Inj. 2019;33(1314):1592-6 A recent systematic literature review of vision rehabilitation interventions for oculomotor deficits in brain injury. Only nine studies met the inclusion criteria; however they demonstrated some efficacy for the use of oculomotor-based intervention in the TBI population.

13. Butler M, Hollestelle E. Visual impairment following acquired brain injury: a survey of occupational therapy practice in New Zealand. New Zeal J Occup Ther. 2017;64:32.

14.• Yoo PY, Scott K, Myszak F, Mamann S, Labelle A, Holmes M, et al. Interventions addressing vision, visual-perceptual impairments following acquired brain injury: a cross-sectional survey. Can J Occup Ther. 2020;87(2):117-26 This article surveyed OTs and their typical practice regarding vision-rehabilitation among adults with acquired brain injury in Canada. Only $3 \%$ of surveyed OT's report using evidence-based treatment for oculomotor impairments.

15. Berger S, Kaldenberg J, Selmane R, Carlo S. Effectiveness of interventions to address visual and visual-perceptual impairments to improve occupational performance in adults with traumatic brain injury: a systematic review. Am J Occup Ther. 2016;70(3): 7003180010p1-7.

16. Simpson-Jones ME, Hunt AW. Vision rehabilitation interventions following mild traumatic brain injury: a scoping review. Disabil Rehabil. 2019;41(18):2206-22 A scoping review of vision rehabilitation interventions following mTBI. Twenty-two studies met inclusion criteria and showed a range of possible intervention strategies.

17. Warren M. Interventions for Adults with Vision Impairment From Acquired Brain Injury. In: Warren M, Barstow EA, editors. Occupational Therapy Interventions For Adults With Low Vision. Bethesda, MD: American Occupational Therapy Association/ AOTA Press; 2011. p. 359-448.
18.• Keilty M, Houston KE, Collins C, Trehan R, Chen YT, Merabet L, et al. Inpatient virtual vision clinic improves access to vision rehabilitation before and during the COVID-19 pandemic. Arch Rehabil Res Clin Transl. 2020;3(1):100100 A descriptive study of a novel video call system that improved the frequency of access to optometry services during inpatient rehabilitation. This pilot study was able to maintain increased access and collaboration with optometry services throughout the COVID-19 pandemic.

19. Herron S. Review of experience with a collaborative eye care clinic in inpatient stroke rehabilitation. Top Stroke Rehabil. 2016;23(1): $67-75$.

20. Hardgrave N, Hatley J, Lewerenz D. Comparing LEA numbers low vision book and Feinbloom visual acuity charts. Optom Vis Sci. 2012;89(11):1611-8.

21. World Health Organization. Consultation on Development of Standards for Characterization of Vision Loss and Visual Functioning 2003 [Available from: https://apps.who.int/iris/ bitstream/handle/10665/68601/WHO PBL 03.91.pdf;jsessionid= D24BFA2F825120F87FE0E4EF89281A68? sequence $=10$ an external site.

22. Warren M. Brain Injury Visual Assessment Battery For Adults Test Manual. visABILITIES Rehab Services: Birmingham; 1998.

23. Berthold-Lindstedt M, Ygge J, Borg K. Visual dysfunction is underestimated in patients with acquired brain injury. J Rehabil Med. 2017;49(4):327-32.

24. Laukkanen H, Scheiman M, Hayes JR. Brain Injury Vision Symptom Survey (BIVSS) Questionnaire. Optom Vis Sci. 2017;94(1):43-50.

25. Warren M. Evaluation and Treatment of Visual Deficits After Brain Injury. In: Pendleton HM, Schultz-Krohn W, editors. Pedretti's Occupational Therapy Practice Skills for Physical Dysfunction. 8th ed. St. Louis: Mosby Elsevier; 2018. p. 594-630.

26. Lotery AJ, Wiggam MI, Jackson AJ, Refson K, Fullerton KJ, Gilmore DH, et al. Correctable visual impairment in stroke rehabilitation patients. Age Ageing. 2000;29(3):221-2.

27. Bertone A, Bettinelli L, Faubert J. The impact of blurred vision on cognitive assessment. J Clin Exp Neuropsychol. 2007;29(5):46776.

28. Johansson J, Berthold Lindstedt M, Borg K. Vision therapy as part of neurorehabilitation after acquired brain injury - a clinical study in an outpatient setting. Brain Inj. 2021;35(1):82-9.

29. American Occupational Therapy Association. Occupational Therapy Practice Framework: Domain and Process (3rd Edition). Am J Occup Ther. 2017;68:S1-S48.

30. Occupational Therapy Code of Ethics. Am J Occup Ther. 2015;69(Suppl 3):6913410030p1-8.

31. Peters M, Price J. The Peters/Price (See to Play) Vision Concussion Protocol: Diagnosis and Treatment. Optometry and Visual Performance. 2015;3(2):126-38.

32. Park UC, Kim SJ, Hwang JM, Yu YS. Clinical features and natural history of acquired third, fourth, and sixth cranial nerve palsy. Eye (Lond). 2008;22(5):691-6.

33. Blanchard S, Chang W-P, Heronema A, Ramcharan D, Stanton K, Stollberg J. Common occupational therapy vision rehabilitation interventions for impaired and low vision associated with brain injury. Optometr Visual Perform. 2016;4:265-74.

34. Rucker JC, Tomsak RL. Binocular diplopia. A practical approach. Neurologist. 2005;11(2):98-110.

35. Politzer TA. Case studies of a new approach using partial and selective occlusion for the clinical treatment of diplopia. NeuroRehabilitation. 1996;6(3):213-7.

36. Gilbert MP, Baker SS. Evaluation and Intervention For Basic and Instrumental Activities of Daily Living. In: Warren M, Barstow EA, editors. Occupational Therapy Interventions For Adults With Low Vision. Bethesda: American Occupational Therapy Association/ AOTA Press; 2011. p. 227-67. 
37. Clark J, Hasselfeld K, Bigsby K, Divine J. Colored glasses to mitigate photophobia symptoms posttraumatic brain injury. J Athl Train. 2017;52(8):725-9.

38. Bowers AR, Meek C, Stewart N. Illumination and reading performance in age-related macular degeneration. Clin Exp Optom. 2001;84(3):139-47.

39. Leigh RJ, Zee DS. Neurology of Eye Movements. 4th ed. New York: Oxford University Press; 2006.

40. Calabrèse A, Owsley C, McGwin G, Legge GE. Development of a reading accessibility index using the MNREAD acuity chart. JAMA Ophthalmol. 2016;134(4):398-405.

41. Nowakowski RW. Basic Optics and Opitcal Devices. In: Warren M, Barstow EA, editors. Occupational Therapy Interventions For Adults With Low Vision. Bethesda: American Occupational Therapy Association/AOTA Press; 2011. p. 75-103.

42. Houston KE, Barrett AM. Patching for diplopia contraindicated in patients with brain injury? Optom Vis Sci. 2017;94(1):120-4.

43. Rowe F. The profile of strabismus in stroke survivors. Eye (Lond). 2010;24(4):682-5.

44. Flanders M, Sarkis N. Fresnel membrane prisms: clinical experience. Can J Ophthalmol. 1999;34(6):335-40.

45. Fox SM, Koons P, Dang SH. Vision rehabilitation after traumatic brain injury. Phys Med Rehabil Clin N Am. 2019;30(1):171-88.

46. Hatt SR, Leske DA, Liebermann L, Holmes JM. Successful treatment of diplopia with prism improves health-related quality of life. Am J Ophthalmol. 2014;157(6):1209-13.

47. Burgess G, Jewell VD. Occupational therapists' perspectives on binocular diplopia in neurorehabilitation: a national survey. NeuroRehabilitation. 2018;42(2):223-33.

48.• Berryman A, Rasavage K, Politzer T, Gerber D. Oculomotor treatment in traumatic brain injury rehabilitation: a randomized controlled pilot trial. Am J Occup Ther. 2020;74(1):7401185050p1-7
A small randomized control study compares a restorative vision exercise protocol to a standard-of-care activity-based intervention for improving oculomotor function following acquired brain injury. Both groups make improvements.

49. Thiagarajan P, Ciuffreda KJ. Effect of oculomotor rehabilitation on accommodative responsivity in mild traumatic brain injury. $\mathrm{J}$ Rehabil Res Dev. 2014;51(2):175-91.

50. Rabinowitz AR, Levin HS. Cognitive sequelae of traumatic brain injury. Psychiatr Clin North Am. 2014;37(1):1-11.

51. Horton JC, Fahle M, Mulder T, Trauzettel-Klosinski S. Adaptation, perceptual learning, and plasticity of brain functions. Graefes Arch Clin Exp Ophthalmol. 2017;255(3):435-47.

52. Kleim JA, Jones TA. Principles of experience-dependent neural plasticity: implications for rehabilitation after brain damage. J Speech Lang Hear Res. 2008;51(1):S225-39.

53. Bogner J, Dijkers M, Hade EM, Beaulieu C, Montgomery E, Giuffrida $\mathrm{C}$, et al. Contextualized treatment in traumatic brain injury inpatient rehabilitation: effects on outcomes during the first year after discharge. Arch Phys Med Rehabil. 2019;100(10): 1810-7 This small study demonstrated that contextualized therapy for those with TBI leads to improved rehabilitation outcomes.

54. Carey L, Walsh A, Adikari A, Goodin P, Alahakoon D, De Silva D, et al. Finding the intersection of neuroplasticity, stroke recovery, and learning: scope and contributions to stroke rehabilitation. Neural Plastic. 2019;2019:5232374.

Publisher's Note Springer Nature remains neutral with regard to jurisdictional claims in published maps and institutional affiliations. 